\title{
Polarization parameters of a spin-one system: Bounds and geometrical representation
}

\author{
G. Ramachandran \\ Department of Physics, Manasagangotri, University of Mysore, Mysore, India 570006 \\ K. S. Mallesh \\ Department of Physics, Yuvaraja's College, University of Mysore, Mysore, India 570005
}

(Received 6 October 1988; revised manuscript received 2 May 1989)

\begin{abstract}
A new geometrical representation for the state of polarization of spin-one particles is suggested which in the limit of mass zero reduces to the well-known Poincare sphere representation for light. A careful consideration of the bounds on the polarization parameters of spin-one resonances produced in parity-conserving processes is also discussed as yet another particular case.
\end{abstract}

\section{INTRODUCTION}

The Poincare sphere representation ${ }^{1}$ for the polarization of light is an old and well-known concept, whose usefulness even today is attested to by several recent applications. ${ }^{2,3}$ Considering that photons are mass zero spin-one particles, it is interesting to examine whether a suitable geometrical representation for the polarization state of massive spin-one particles could be given such that it reduces to the Poincare representation in the case of massless particles. Here we show that it is indeed possible.

Following the Madison convention ${ }^{4}$ (which is identical with the earlier usage by Ramachandran and Umerjee ${ }^{5}$ ), the density matrix $\rho$ for massive spin-one particles is given explicitly by

$$
\rho=\frac{\operatorname{Tr} \rho}{3}\left(\begin{array}{ccc}
1+\sqrt{3 / 2} t_{0}^{1}+\frac{1}{\sqrt{2}} t_{0}^{2} & \sqrt{3 / 2}\left(t_{-1}^{1}+t_{-1}^{2}\right) & \sqrt{3} t_{-2}^{2} \\
-\sqrt{3 / 2}\left(t_{1}^{1}+t_{1}^{2}\right) & 1-\sqrt{2} t_{0}^{2} & \sqrt{3 / 2}\left(t_{-1}^{1}-t_{-1}^{2}\right) \\
\sqrt{3} t_{2}^{2} & -\sqrt{3 / 2}\left(t_{1}^{1}-t_{1}^{2}\right) & 1-\sqrt{3 / 2} t_{0}^{1}+\frac{1}{\sqrt{2}} t_{0}^{2}
\end{array}\right)
$$

in terms of the spherical tensor parameters $t_{q}^{k}$ of rank $k=1,2$ and $q=-k$ to $+k$. The rows and columns are labeled by the states $|1, m\rangle ; m=+1,0,-1$. The general structure (1) for $\rho$ assumes simplified forms for specialized choices of the Cartesian axes in the real threedimensional space. (1) If we choose the $z$ axis to be along the direction of vector polarization, then $t_{ \pm 1}^{1}=0$; such a frame may be referred to as a Lakin frame. ${ }^{6}$ Moreover, by a suitable rotation about the $z$ axis one can render $\operatorname{Im}\left(t_{1}^{2}\right)=0$ and $\operatorname{Re}\left(t_{1}^{2}\right) \geq 0$. We shall refer to the Lakin frame in which these two further conditions are satisfied as the special Lakin frame (SLF). This is a convenient frame to work with as long as the vector polarization is nonzero. (2) Observing that the parameters $t_{q}^{2}$ representing the tensor polarization are effectively equivalent to a real symmetric second-rank Cartesian tensor ${ }^{4,5}$ $p_{\alpha \beta}=2 Q_{\alpha \beta}$, which defines a set of the principal axes; one can choose the Cartesian axes to be along the principal axes. Such a frame has been referred to ${ }^{7}$ as a principal axis of alignment frame (PAAF), where $\left(t_{ \pm 1}^{2}\right)^{A}$ $=\operatorname{Im}\left(t_{2}^{2}\right)^{A}=0$, since a polarized spin system with a zero vector polarization, but with a nonzero tensor polarization, is usually referred to as an aligned system. The three eigenstates of any aligned system can conveniently be looked upon ${ }^{7}$ as the $|j m\rangle$ state with $m=0$, with the axis of quantization chosen, respectively, along the $X, Y$, $Z$ directions defined by the principal axes. The states were therefore denoted by $\left|x_{A}\right\rangle,\left|y_{A}\right\rangle$, and $\left|z_{A}\right\rangle$ earlier. Moreover, if $\lambda_{i} ; i=1,2,3$ denote the eigenvalues of $\rho$ for an aligned system, it follows that if any two eigenvalues are equal and the two corresponding eigenstates are designated as $\left|x_{A}\right\rangle$ and $\left|y_{A}\right\rangle$, the system is "oriented"8 with the $z_{A}$ axis being the axis of orientation. If all the $\lambda_{i}$ are different, the aligned system is nonoriented and is characterized by two independent axes ${ }^{9}$ in the threedimensional configuration space.

In view of the above, it is advantageous for our purposes here to define a special frame (SF) which is identical with SLF for systems with nonzero vector polarization and coincides with a PAAF for aligned systems.

The density matrix description for light, i.e., massless spin-one particles is well known ${ }^{10,11}$ and is given by

$$
\rho=\frac{1}{2}\left(\begin{array}{cc}
1+p_{1} & p_{2}-i p_{3} \\
p_{2}+i p_{3} & 1-p_{1}
\end{array}\right),
$$

where we have put $\operatorname{Tr} \rho=1$ and labeled the rows and columns by the plane-polarized states along $x$ and $y$ 
directions, the direction of propagation is along $z$ axis. The parameters $p_{1}, p_{2}$, and $p_{3}$ denote the well-known Stokes's parameters: ${ }^{12}$ choosing $p_{1}, p_{2}, p_{3}$ along the three axes of a Cartesian frame, the state of polarization is represented by a vector $\mathbf{p}$. For pure states, $\operatorname{Tr}\left(\rho^{2}\right)=(\operatorname{Tr} \rho)^{2}=1$, which gives $p_{1}^{2}+p_{2}^{2}+p_{3}^{2}=1$ so that the tip of the vector $\mathbf{p}$ with components $p_{1}, p_{2}$, and $p_{3}$ lies on the surface of a unit sphere which is the well-known Poincare sphere. ${ }^{1}$ For mixture states $\operatorname{Tr}\left(\rho^{2}\right)<1$, and consequently the state of partially polarized light may be represented by a point within the sphere.

Expressing the density matrix $\rho$ given by (2) in the $3 \times 3$ form with rows and columns labeled by $|1,+1\rangle$, $|1,0\rangle$, and $|1,-1\rangle$ states, we have

$$
\rho=\frac{1}{2}\left(\begin{array}{ccc}
1-p_{3} & 0 & -p_{1}-i p_{2} \\
0 & 0 & 0 \\
-p_{1}+i p_{2} & 0 & 1+p_{3}
\end{array}\right)
$$

Here the $m= \pm 1$ states (which are the only ones available for physical photons) are defined through

$$
|1, \pm 1\rangle=\mp \frac{1}{\sqrt{2}}\left(\hat{\boldsymbol{\epsilon}}_{x} \pm i \hat{\boldsymbol{\epsilon}}_{y}\right)
$$

where $\hat{\boldsymbol{\epsilon}}_{x}, \hat{\boldsymbol{\epsilon}}_{y}$ denote the unit vectors along the $x$ and $y$ directions. If the electromagnetic wave is represented by

$$
\exp \left[-2 \pi i\left[v t-\frac{z}{\lambda}\right]\right]
$$

the $|1,+1\rangle$ and $|1,-1\rangle$ states defined above correspond, respectively, to the conventional ${ }^{13}$ left and right circular polarizations.

\section{GEOMETRICAL REPRESENTATION}

To arrive at a geometrical representation for the state of polarization of massive spin-one particles, let us compare Eqs. (1) and (3). We notice that

$$
t_{ \pm 1}^{1}=t_{ \pm 1}^{2}=0
$$

for light. In other words, the choice of the $z$ axis to be the direction of propagation (and the absence of the longitudinal state of polarization) immediately implies that we are working in a SLF. The comparison further leads to the identification

$$
\begin{aligned}
& -\frac{2}{\sqrt{3}} \operatorname{Re}\left(t_{2}^{2}\right)=p_{1}, \\
& +\frac{2}{\sqrt{3}} \operatorname{Im}\left(t_{2}^{2}\right)=p_{2}, \\
& -\sqrt{2 / 3} t_{0}^{1}=p_{3},
\end{aligned}
$$

$$
t_{0}^{2}=\frac{1}{\sqrt{2}} .
$$

Irrespective of whether a spin-one system is massive or massless, the condition that the eigenvalues of $\rho$ must be non-negative implies that all its principal minors should also be nonnegative. ${ }^{14}$ From this result of the matrix theory, ${ }^{15}$ the positive semidefiniteness property of the density matrix leads to

$$
\begin{aligned}
& 1+\sqrt{3 / 2} t_{0}^{1}+\frac{1}{\sqrt{2}} t_{0}^{2} \geq 0, \\
& 1-\sqrt{2} t_{0}^{2} \geq 0, \\
& 1-\sqrt{3 / 2} t_{0}^{1}+\frac{1}{\sqrt{2}} t_{0}^{2} \geq 0, \\
& \left(1+\sqrt{3 / 2} t_{0}^{1}+\frac{1}{\sqrt{2}} t_{0}^{2}\right)\left(1-\sqrt{2} t_{0}^{2}\right)-\frac{3}{2}\left|t_{1}^{2}\right|^{2} \geq 0, \\
& \left(1-\sqrt{3 / 2} t_{0}^{1}+\frac{1}{\sqrt{2}} t_{0}^{2}\right)\left(1-\sqrt{2} t_{0}^{2}\right)-\frac{3}{2}\left|t_{1}^{2}\right|^{2} \geq 0, \\
& \left(1+\frac{1}{\sqrt{2}} t_{0}^{2}\right)^{2}-\frac{3}{2}\left(t_{0}^{1}\right)^{2}-3\left|t_{2}^{2}\right|^{2} \geq 0, \\
& \left(1-\sqrt{2} t_{0}^{2}\right)\left[\left(1+\frac{1}{\sqrt{2}} t_{0}^{2}\right)^{2}-\frac{3}{2}\left(t_{0}^{1}\right)^{2}-3\left|t_{2}^{2}\right|^{2}\right] \\
& -3 \operatorname{Re}\left(t_{1}^{2}\right)^{2}\left[1+\frac{1}{\sqrt{2}} t_{0}^{2}+\sqrt{3} \operatorname{Re}\left(t_{2}^{2}\right)\right] \geq 0,
\end{aligned}
$$

which imply together

$$
\begin{aligned}
& -\sqrt{2} \leq t_{0}^{2} \leq \frac{1}{\sqrt{2}}, \\
& -\sqrt{3 / 2} \leq t_{0}^{1} \leq \sqrt{3 / 2} \\
& 0 \leq\left(t_{0}^{1}\right)^{2}+2\left[\operatorname{Re}\left(t_{2}^{2}\right)\right]^{2}+2\left[\operatorname{Im}\left(t_{2}^{2}\right)\right]^{2} \\
& \left.\leq \frac{2}{3} \cdot 1+\frac{1}{\sqrt{2}} t_{0}^{2}\right]^{2}, \\
& 0 \leq\left[\operatorname{Re}\left(t_{1}^{2}\right)\right]^{2} \leq \frac{2}{3}\left[1+\sqrt{3 / 2} t_{0}^{1}+\frac{1}{\sqrt{2}} t_{0}^{2}\right)\left(1-\sqrt{2} t_{0}^{2}\right), \\
& 0 \leq\left[\operatorname{Re}\left(t_{1}^{2}\right)\right]^{2} \leq \frac{2}{3}\left[1-\sqrt{3 / 2} t_{0}^{1}+\frac{1}{\sqrt{2}} t_{0}^{2}\right)\left(1-\sqrt{2} t_{0}^{2}\right) \\
& 0 \leq \operatorname{Re}\left(t_{1}^{2}\right) \leq Q
\end{aligned}
$$

where

$$
Q=\left\{\left[\left(1+\frac{1}{\sqrt{2}} t_{0}^{2}\right]^{2}-\frac{3}{2}\left(t_{0}^{1}\right)^{2}-3\left|t_{2}^{2}\right|^{2}\right]\left(1-\sqrt{2} t_{0}^{2}\right) / 3\left[1+\frac{1}{\sqrt{2}} t_{0}^{2}+\sqrt{3} \operatorname{Re}\left(t_{2}^{2}\right)\right]\right\}^{1 / 2} \ldots
$$


with the positive root taken in $(7 \mathrm{~g})$ since, by definition, $\operatorname{Re}\left(t_{1}^{2}\right) \geq 0$ in SF.

If we define parameters $p_{1}, p_{2}$, and $p_{3}$ even for massive spin-one particles using the same equations $(5 b)-(5 d)$, it is clear that

$$
p^{2}=p_{1}^{2}+p_{2}^{2}+p_{3}^{2} \leq R^{2}=\left(\frac{2}{3}\right)^{2}\left(1+\frac{1}{\sqrt{2}} t_{0}^{2}\right)^{2} .
$$

Moreover, any arbitrary spin state of massive spin-one particles is completely specified in SF if we give $\operatorname{Re}\left(t_{1}^{2}\right)$ in addition to $p_{1}, p_{2}, p_{3}$, and $t_{0}^{2}$.

Let us, therefore, represent the possible values of $\operatorname{Re}\left(t_{1}^{2}\right)$ ranging from $O$ to $Q$ on a real line represented horizontally as in Fig. 1. We then draw a sphere of radius $R$ such that it touches the horizontal line at the specified value of the parameter $\operatorname{Re}\left(t_{1}^{2}\right)$. choosing a Cartesian frame $0 X, O Y, O Z$ (coinciding with SF), and with the center of the sphere as the origin, we draw a vector $\mathbf{p}$ with components $p_{1}, p_{2}$, and $p_{3}$. The tip of this vector naturally lies on or within the sphere in view of (8). It is clear that the figure geometrically represents any arbitrary state of polarization of the spin-one particles irrespective of their mass. It may be noted in passing that the directions $0 X, 0 Y$, and $0 Z$ (along which $p_{1}, p_{2}$, and $p_{3}$ are marked off) specify the SF in the configuration space. However, $p_{1}, p_{2}$, and $p_{3}$ are related to $\operatorname{Re}\left(t_{2}^{2}\right), \operatorname{Im}\left(t_{2}^{2}\right)$, and $t_{0}^{1}$, which, together, do not transform under rotations like the Cartesian components of a vector. Likewise, it should be remembered that $\operatorname{Re}\left(t_{1}^{2}\right)$, which is represented along a line, transforms as a component of an irreducible tensor of rank 2 under rotations and thus it is neither a vector nor a scalar in the configuration space.

In the limit of mass zero particles it is clear from (5a) that the sphere will always touch the line at $\operatorname{Re}\left(t_{1}^{2}\right)=0$ and consequently the line becomes redundant. Also, Eqs. (5e) and (8) imply that the radius $R$ of the sphere is fixed

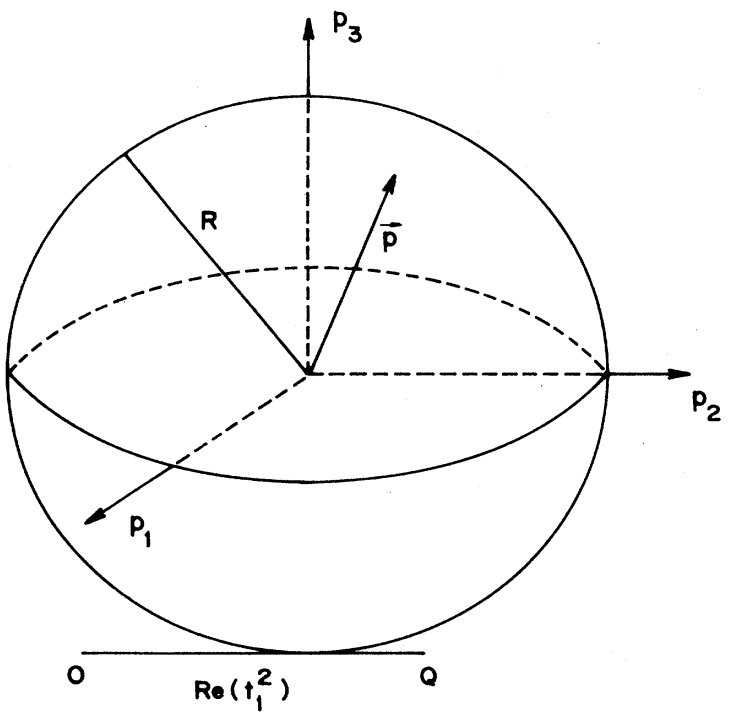

FIG. 1. Geometrical representation of the state of polarization of a spin-one system. and equals 1. Thus, the vector $\mathbf{p}$ on or within the unit sphere, by itself, completely represents any arbitrary state of polarization of the zero mass particle.

The geometrical representation outlined above, when specialized to mass zero particles, leads. to the following additional features for the conventional Poincare sphere. It is capable of representing any arbitrary state of partially polarized light; moreover, the $z$ axis gives the direction of propagation in space, and the two states of plane polarization which are chosen as the basis states are along $X$ and $Y$.

\section{SPECIAL CASES}

We now discuss some special cases of interest and determine the corresponding restricted domains of the state of polarization in our geometrical representation.

\section{A. Case 1: Purely vector polarized system}

The only nonzero parameter in SF of such a spin system is $t_{0}^{1}$. Consequently, the vector $\mathrm{p}$ lies along $p_{3}$ or $z$ axis on or inside a sphere of radius $R=\frac{2}{3}$ with the sphere touching the horizontal line at $\operatorname{Re}\left(t_{1}^{2}\right)=0$.

\section{B. Case 2: Aligned system}

Since the only nonzero parameters in this case are $t_{0}^{2}$ and $\operatorname{Re}\left(t_{2}^{2}\right)$, aligned systems are represented by the vector p lying along $p_{1}$ or the $x$ axis with the sphere touching the horizontal line at $\operatorname{Re}\left(t_{1}^{2}\right)=0$.

\section{Case 3: Oriented systems}

If the system is aligned and oriented and the principal axes are properly designated such that $\left|x_{A}\right\rangle$ and $\left|y_{A}\right\rangle$ represent the eigenstates with a common eigenvalue, $\operatorname{Re}\left(t_{2}^{2}\right)$ is also zero. Consequently, such a state of polarization is represented by the center of the sphere which touches the horizontal line at $\operatorname{Re}\left(t_{1}^{2}\right)=0$.

However, an oriented system can also possess nonzero vector polarization. In that case the parameters $t_{ \pm 1}^{1}, t_{ \pm 1}^{2}$, and $t_{ \pm 2}^{2}$ will all be zero in SF, and consequently the spin state of such an oriented system is specified in SF by $t_{0}^{1}$ and $t_{0}^{2}$ only. From this it follows that the polarization states in this case are represented by points on $p_{3}$ or the $z$ axis on or inside the sphere which touches the line at $\operatorname{Re}\left(t_{1}^{2}\right)=0$.

\section{Case 4: Pure states}

If the density matrix $\rho$ represents a pure state then it should be idempotent, i.e.,

$$
\rho^{2}=\rho
$$

and all its principal minors of dimension greater than 1 will vanish. Thus, we have

$\left(t_{0}^{1}\right)^{2}+2\left[\operatorname{Re}\left(t_{2}^{2}\right)\right]^{2}+2\left[\operatorname{Im}\left(t_{2}^{2}\right)\right]^{2}=\frac{2}{3}\left(1+\frac{1}{\sqrt{2}} t_{0}^{2}\right)^{2}$, 
$\left[\operatorname{Re}\left(t_{1}^{2}\right)\right]^{2}=\frac{2}{3}\left[1+\sqrt{3 / 2} t_{0}^{1}+\frac{1}{\sqrt{2}} t_{0}^{2}\right)\left(1-\sqrt{2} t_{0}^{2}\right)$

$\left[\operatorname{Re}\left(t_{1}^{2}\right)\right]^{2}=\frac{2}{3}\left[1-\sqrt{3 / 2} t_{0}^{1}+\frac{1}{\sqrt{2}} t_{0}^{2}\right)\left(1-\sqrt{2} t_{0}^{2}\right)$

$\operatorname{Re}\left(t_{1}^{2}\right)^{2}\left[1+\frac{1}{\sqrt{2}} t_{0}^{2}+\sqrt{3} \operatorname{Re}\left(t_{2}^{2}\right)\right]=0$.

From Eqs. (10b) and (10c) we have

$$
\begin{aligned}
\operatorname{Re}\left(t_{1}^{2}\right)^{2} & =\frac{2}{3}\left[1+\sqrt{3 / 2} t_{0}^{1}+\frac{1}{\sqrt{2}} t_{0}^{2}\right]\left(1-\sqrt{2} t_{0}^{2}\right) \\
& =\frac{2}{3}\left[1-\sqrt{3 / 2} t_{0}^{1}+\frac{1}{\sqrt{2}} t_{0}^{2}\right]\left(1-\sqrt{2} t_{0}^{2}\right),
\end{aligned}
$$

which implies either $t_{0}^{2}=1 / \sqrt{2}$ or $t_{0}^{1}=0$. We therefore have two categories of pure states: (a)

$$
t_{0}^{2}=\frac{1}{\sqrt{2}}
$$

For this value of $t_{0}^{2}$ we see from (10b) and 10(c) that $\operatorname{Re}\left(t_{1}^{2}\right)=0$ and, moreover, Eq. (10a) becomes

$$
\left(t_{0}^{1}\right)^{2}+2\left[\operatorname{Re}\left(t_{2}^{2}\right)\right]^{2}+2\left[\operatorname{Im}\left(t_{2}^{2}\right)\right]^{2}=\frac{3}{2},
$$

which leads to

$$
p^{2}=p_{1}^{2}+p_{2}^{2}+p_{3}^{2}=1 \text {. }
$$

Thus, the pure states in this case correspond to points on the surface of the unit sphere which touches the line at $\operatorname{Re}\left(t_{1}^{2}\right)=0$.

The density matrix $\rho$ in this case has the explicit form

$$
\rho=\frac{1}{3}\left(\begin{array}{ccc}
\frac{3}{2}+\sqrt{3 / 2} t_{0}^{1} & 0 & \sqrt{3} t_{-2}^{2} \\
0 & 0 & 0 \\
\sqrt{3} t_{2}^{2} & 0 & \frac{3}{2}-\frac{\sqrt{3}}{2} t_{0}^{1}
\end{array}\right),
$$

which immediately shows that the pure state must be a linear combination of the $|1, \pm 1\rangle$ states. It is to be noted, in particular, that if $t_{ \pm 2}^{2}=0$, then we have by virtue of (12) that $t_{0}^{1}= \pm \sqrt{3 / 2}$, and these two cases correspond simply to the pure states $|1, \pm 1\rangle$, and are represented, respectively, by the south and north poles on the unit sphere in Fig. 1. (b)

$$
t_{0}^{1}=0 \text {. }
$$

In this case obviously the system is aligned and consequently we consider $\rho$ in PAAF, where $t_{ \pm 1}^{2}=0$ and $\operatorname{Im}\left(t_{2}^{2}\right)=0$. Equations (10b) and (10c) imply that either $t_{0}^{2}=1 / \sqrt{2}$ or $-\sqrt{2}$. Equation (10a) then shows that $\operatorname{Re}\left(t_{2}^{2}\right)$ can only assume values $\pm \sqrt{3 / 2}$ when $t_{0}^{2}=1 / \sqrt{2}$ and zero when $t_{0}^{2}=-\sqrt{2}$. Looking at the explicit form of the density matrix

$$
\rho=\frac{1}{3}\left(\begin{array}{ccc}
1+\frac{1}{\sqrt{2}} t_{0}^{2} & 0 & \sqrt{3} \operatorname{Re}\left(t_{2}^{2}\right) \\
0 & 1-\sqrt{2} t_{0}^{2} & 0 \\
\sqrt{3} \operatorname{Re}\left(t_{2}^{2}\right) & 0 & 1+\frac{1}{\sqrt{2}} t_{0}^{2}
\end{array}\right)
$$

we readily see that $t_{0}^{2}=-\sqrt{2}$ and $\operatorname{Re}\left(t_{2}^{2}\right)=0$ correspond simply to the state $|1,0\rangle$, while

$$
t_{0}^{2}=1 / \sqrt{2} \text { and } \operatorname{Re}\left(t_{2}^{2}\right)=\mp \sqrt{3 / 2}
$$

correspond, respectively, to the pure states

$$
\left|x_{A}\right\rangle=-\frac{[|1,+1\rangle-|1,-1\rangle]}{\sqrt{2}}
$$

and

$$
\left|y_{A}\right\rangle=\frac{i[|1,+1\rangle+|1,-1\rangle]}{\sqrt{2}} .
$$

Thus, the only pure states which are characterized by $t_{0}^{1}=0$ are the three eigenstates designated as $\left|x_{A}\right\rangle,\left|y_{A}\right\rangle$, and $\left|z_{A}\right\rangle$ in Ref. 5. $\left|x_{A}\right\rangle$ and $\left|y_{A}\right\rangle$ are represented by the diametrically opposite points on the unit sphere along $p_{1}$ or the $x$ axis, while in the case of the state $\left|z_{A}\right\rangle$, the sphere reduces to a point. In all the cases $\operatorname{Re}\left(t_{1}^{2}\right)=0$, the point at which the sphere touches the line.

Thus, all pure states are characterized by $\operatorname{Re}\left(t_{1}^{2}\right)=0$ in our special frame and are either represented by points on a unit sphere or by a sphere with radius zero. The former are linear combinations of $|1, \pm 1\rangle$ states, while the latter corresponds uniquely to the $|1,0\rangle$ state. The latter situation does not arise in the case of massless particles for which the longitudinal state does not exist and the unit sphere is, in fact, the Poincare sphere where diametrically opposite points are well known to represent mutually orthogonal elliptically polarized states.

Even in the case of massive spin-one particles such a statement can be made. Consider two orthogonal pure states $\left|\psi_{1}\right\rangle$ and $\left|\psi_{2}\right\rangle$ with

$$
\left|\psi_{1}\right\rangle=\frac{1}{\left(a^{2}+b^{2}\right)^{1 / 2}}[a|1,+1\rangle+i b|1,-1\rangle],
$$

and

$$
\left|\psi_{2}\right\rangle=\frac{1}{\left(a^{2}+b^{2}\right)^{1 / 2}}[i b|1,+1\rangle+a|1,-1\rangle] .
$$

Then the corresponding density matrices take the form

$$
\rho_{1}=\frac{1}{a^{2}+b^{2}}\left(\begin{array}{ccc}
a^{2} & 0 & -i a b \\
0 & 0 & 0 \\
i a b & 0 & b^{2}
\end{array}\right]
$$

and

$$
\rho_{2}=\frac{1}{a^{2}+b^{2}}\left[\begin{array}{ccc}
b^{2} & 0 & +i a b \\
0 & 0 & 0 \\
-i a b & 0 & a^{2}
\end{array}\right] .
$$

Comparing (20) and (21) with (14) we see that

$$
t_{0}^{1}(2)=-t_{0}^{1}(1), \quad t_{2}^{2}(2)=-t_{2}^{2}(1),
$$


and consequently they are represented on the unit sphere by diametrically opposite points. It is interesting to point out that the condition $\rho_{1} \rho_{2}=0$ for orthogonal pure states is readily satisfied by (20) and (21).

\section{E. Case 5: Spin-one resonances produced in parity-conserving reactions}

Another special case of interest concerns the state of polarization of spin-one particles or resonances produced in parity-conserving reactions ${ }^{16,17}$ where $t_{ \pm 1}^{1}=t_{ \pm 1}^{2}=0$ in a frame of reference where the $z$ axis is chosen to be normal to the reaction plane. This frame corresponds to our special frame with a further condition that $\operatorname{Re}\left(t_{1}^{2}\right)=0$ on account of parity conservation. Minnaert has used the condition

$$
2 \operatorname{Tr} \rho^{3}-3 \operatorname{Tr} \rho^{2}+1 \geq 0,
$$

in addition to

$$
\operatorname{tr} \rho^{2} \leq 1
$$

in discussing the domains of the variations of the parameters $t_{q}^{k}$. We only have to point out that condition (23) is really a single condition obtained by adding the three individual conditions $(6 \mathrm{~d})-(6 f)$ enunciated here and leads to

$$
\left(t_{0}^{1}\right)^{2}+\left(t_{0}^{2}\right)^{2}+2\left|t_{2}^{2}\right|^{2} \leq 2,
$$

which is nothing but his Eq. (6a). (Note that the multipole parameters used by Minnaert are normalized differently from the Madison convention.) This is used to define an ellipsoid in a three-dimensional space where the coordinates are $t_{0}^{1}, t_{0}^{2}$, and $\left|t_{2}^{2}\right|$. However, we have to note that the ellipsoid cannot be a complete one since $\left|t_{2}^{2}\right| \geq 0$. Moreover, one cannot specify $t_{ \pm 2}^{2}$ given $\left|t_{2}^{2}\right|$ alone. On the other hand, the geometrical representation given here completely specifies $t_{ \pm 2}^{2}$ since $\operatorname{Re}\left(t_{2}^{2}\right)$ and $\operatorname{Im}\left(t_{2}^{2}\right)$ are represented along two orthogonal coordinate axes. Condition (22) used by Minnaert is already implied in our discussion through the use of (6f). Since $\operatorname{Re}\left(t_{ \pm 1}^{2}\right)=0$, the polarization domain in this special case is completely specified by points which are on or within the sphere of radius $R$, the sphere touching the line at $\operatorname{Re}\left(t_{1}^{2}\right)=0$.

\section{F. Case 6: Comparison with Robson's treatment}

Before carrying out a comparison with Robson's ${ }^{18}$ treatment we must recall that a spin-one system can either be oriented (i.e., monoaxial) or nonoriented (i.e., multiaxial - either biaxial or triaxial) in general.

The diagram 4.1 of Robson's book provides a description of the bounds only on $t_{0}^{1}$ and $t_{0}^{2}$. If the system happens to be oriented, and further if the $z$ axis is chosen parallel to the axis of orientation, then $t_{0}^{1}$ and $t_{0}^{2}$ are the only nonzero tensor parameters and can be related to the statistical weights. Only in such a situation can Fig. 4.1 be considered to provide a complete description of the bounds. By expressing $\rho$ as a sum of three (not necessarily unique) density matrices $\rho_{i}(i=1,2,3)$ corresponding to pure unnormalized states $\psi_{i}$ where

$$
\begin{aligned}
& \left|\psi_{1}\right\rangle=a_{1}|+1\rangle+a_{2}|0\rangle+a_{3}|-1\rangle, \\
& \left|\psi_{2}\right\rangle=b_{1}|+1\rangle+b_{3}|-1\rangle, \\
& \left|\psi_{3}\right\rangle=d_{2}|0\rangle,
\end{aligned}
$$

Robson has shown that $\left|t_{1}^{1}\right|,\left|t_{1}^{2}\right|$, and $\left|t_{2}^{2}\right|$ have $\sqrt{3 / 2}$ as their upper limit. It should be noted, however, that the upper limit will be less than $\sqrt{3 / 2}$ in certain cases. For example, when $t_{0}^{1}=0, t_{0}^{2}=1 / \sqrt{2}$ we have $\left|t_{2}^{2}\right|<1 / \sqrt{2}$ and $\operatorname{Re}\left(t_{1}^{2}\right)=0$. Our Eqs. (7c) $-(7 f)$ also contain this additional information and consequently provide more restrictive bounds. However, it should be noted that our bounds viz., Eqs. (6) and (7), are derived in the special frame SF, where $t_{1}^{1}$ and $\operatorname{Im} t_{1}^{2}$ are zero. Further, the geometric representation suggested in this paper is valid in general, whether the system is oriented or nonoriented.

As compared to the geometric representation suggested in Ref. 7 (which is also generally valid) the present one is far more interesting in that it is analogous to the celebrated Poincare representation and contains complete information on the spin-one system. The axes $0 X, 0 Y$, and $O Z$ specify the frame $S F$ in the configuration space and the parameters $p_{1}, p_{2}$, and $p_{3}$ (which are, purely for economy, marked along $0 X, 0 Y$, and $0 Z$, but do not constitute a vector in the configuration space) give us $\operatorname{Re} t_{2}^{2}, \operatorname{Im} t_{2}^{2}$, and $t_{0}^{1}$, respectively, while the radius of the sphere specifies $t_{0}^{2}$ and the point at which the sphere touches the tangent line marks off $\operatorname{Re}\left(t_{1}^{2}\right)$ thereby specifying all the nonzero $t_{q}^{k}$, s in the SF.
${ }^{1}$ M. Born and E. Wolf, Principles of Optics (Pergamon, London, 1959), p. 31.

${ }^{2}$ M. Blume and Doon Gibbs, Phys. Rev. B 37, 1779 (1988).

${ }^{3}$ M. Brunel and F. de Bergevin, Acta Crystallogr. Sect. A 37, 344 (1981).

${ }^{4}$ S. E. Darden, in Proceedings of the International Conference on Polarization Phenomena in Nuclear Reactions, edited by $\mathbf{H}$. $\mathbf{H}$. Barschall and W. Haeberli (University of Wisconsin Press, Madison, Wisconsin, 1971), p. 39.

${ }^{5}$ G. Ramachandran and R. K. Umerjee, Nucl. Phys. 54, 665
(1964).

${ }^{6}$ W. Lakin, Phys. Rev. 98, 139 (1955).

${ }^{7}$ G. Ramachandran, K. S. Mallesh, and V. Ravishankar, J. Phys. G 10, L164 (1984).

${ }^{8}$ G. Ramachandran and K. S. Mallesh, Nucl. Phys. A422, 327 (1984).

${ }^{9}$ G. Ramachandran and V. Ravishankar, J. Phys. G 12, L143 (1986).

${ }^{10}$ U. Fano, Rev. Mod. Phys. 29, 74 (1957).

${ }^{11}$ W. H. McMaster, Rev. Mod. Phys. 33, 8 (1961). 
${ }^{12}$ G. G. Stokes, Trans. Cambridge Philos. Soc. 9, 399 (1852).

13J. D. Jackson, Classical Electrodynamics (Wiley Eastern, New Delhi, 1978), p. 274.

${ }^{14}$ W. Band and J. L. Park, J. Math. Phys. 14, 551 (1973).

${ }^{15}$ F. R. Gantmacher, The Theory of Matrices (Chelsea, New
York, 1959), p. 337.

${ }^{16}$ R. H. Dalitz, Annu. Rev. Nucl. Sci. 13, 339 (1963).

${ }^{17}$ P. Minnaert, Phys. Rev. Lett. 16, 672 (1966).

${ }^{18}$ B. A. Robson, Theory of Polarization Phenomena (Clarendon, Oxford, 1974), p. 61. 